\title{
Quantitative analysis of directional spontaneous emission spectra from light sources in photonic crystals
}

\author{
Ivan S. Nikolaev, ${ }^{*}$ Peter Lodahl, and Willem L. Vos \\ Complex Photonic Systems (COPS), Department of Science and Technology, and MESA Institute of Nanotechnology, \\ University of Twente, P.O. Box 217, 7500 AE Enschede, The Netherlands ${ }^{\dagger}$
}

(Received 26 July 2004; published 25 May 2005)

\begin{abstract}
We have performed angle-resolved measurements of spontaneous-emission spectra from laser dyes and quantum dots in opal and inverse opal photonic crystals. Pronounced directional dependencies of the emission spectra are observed: angular ranges of strongly reduced emission adjoin with angular ranges of enhanced emission. It appears that emission from embedded light sources is affected both by the periodicity and by the structural imperfections of the crystals: the photons are Bragg diffracted by lattice planes and scattered by unavoidable structural disorder. Using a model comprising diffuse light transport and photonic band structure, we quantitatively explain the directional emission spectra. This work provides detailed understanding of the transport of spontaneously emitted light in real photonic crystals, which is essential in the interpretation of quantum optics in photonic-band-gap crystals and for applications wherein directional emission and total emission power are controlled.
\end{abstract}

DOI: 10.1103/PhysRevA.71.053813

PACS number(s): 42.50.Nn, 42.70.Qs, 78.67.Hc, 42.50.Ct

\section{INTRODUCTION}

Photonic crystals attract much attention both in academia and in industry because they offer exciting ways of manipulating photons [1]. Periodic variations of the refractive index in photonic crystals on a length scale of the wavelength of light cause optical Bragg diffraction and organize the photon dispersion relation in bands, analogous to electron bands in semiconductors [2-4]. Frequency windows, called stop bands, appear in which there are no modes for certain propagation directions. Photonic structures already serve as a base for controlling the propagation of light. Of even greater interest are three-dimensional (3D) photonic crystals which offer the opportunity of a photonic band gap (PBG) - a frequency range where no modes exist at all. The existence of a gap in the density of photonic modes leads to interesting quantum-optical phenomena such as spontaneous emission inhibition and light localization [2-6].

Control over the radiative decay rate of spontaneous emission is of keen interest for applications, and therefore emission properties of sources such as atoms, dyes, and quantum dots are intensively investigated. According to Fermi's golden rule, this decay rate is proportional to the local radiative density of states (LDOS) that counts the number of electromagnetic modes wherein photons can be emitted at a specific location of the source. In 3D photonic crystals pronounced variations of the LDOS with frequency are predicted even in the absence of a PBG $[7,8]$, which give rise to angle-independent variations of the total emission. Recently LDOS effects on spontaneous emission have been experimentally demonstrated: Using inverse opal photonic crystals considerable variations of the emission rates in large bandwidths were obtained in both continuous-wave $(\mathrm{cw})$ total-

\footnotetext{
*Electronic address: i.nikolaev@amolf.nl

${ }^{\dagger}$ URL: www.photonicbandgaps.com
}

emission power experiments [9] and time-resolved lifetime experiments [10]. While lifetime experiments provide a direct measurement of decay rates, it is important to quantitatively interpret concomitant emission spectra, for instance, to confirm that light sources inside the crystal are probed. $\mathrm{Cw}$ experiments, on the other hand, rely on a comparison of angle-integrated spectra with a homogeneous medium. In the latter case a complete understanding of all angle-dependent effects, that is, Bragg diffraction on the propagation of light is crucial. In this paper such a quantitative analysis is presented.

In contrast to decay rates, emission spectra of sources embedded in photonic crystals are strongly directional [11-16]. Particular frequency ranges of the spectra are suppressed in certain directions forming stop bands, whose center frequencies and widths are described by the photon dispersion relation. Besides Bragg diffraction, which is an effect of the order of the periodic structure, light propagating inside the structure also experiences disorder: polydispersity, roughness, and misarrangements of the building blocks [17]. This unavoidable disorder affects the interference-induced properties of photonic crystals. Previous work on the effect of disorder on spontaneous emission includes the realization that disorder determines the depth of stop bands $[13,15]$. Furthermore, the first observations of enhanced emission in the range of first- and second-order stop bands were also related to disorder-induced redistribution of emitted photons [18]. While the propagation of light from external light sources has been studied in great detail [19-21], a quantitative explanation of the behavior of light emission from internal sources has lacked sofar.

Here we present strongly frequency-dependent angular distributions of spontaneous emission from a laser dye in polystyrene opals and from quantum dots in titania inverse opals in the frequency range around the first-order Bragg diffraction ( $L$-gap). We compare the data to a theoretical model that unifies effects of structural disorder and photonic 
crystal properties [21]. Angle-dependent internal reflection due to the photonic gaps plays a key role in our model. The theory quantitatively explains both the enhancement and the reduction of light along certain propagation directions that were observed experimentally. The excellent agreement confirms that the propagation of light in a photonic crystal is well understood for frequencies around the $L$-gap. Furthermore, we show that by analyzing the exit emission distributions, one can reveal stop bands in the quantum-dot spectra. Such an analysis should be carefully performed before any quantum-optical experiments since it unambiguously proves the effect of the photonic crystal on emission. We finally discuss the applicability of photonic crystals for improvement of the emission efficiency of light sources.

\section{EXPERIMENTAL DETAILS}

\section{A. Samples}

We have studied emission from dyes in polystyrene opals and from quantum dots in titania inverse opals. The polystyrene opals are fcc crystals of close-packed polystyrene spheres prepared from a colloidal suspension by selfassembly. The titania inverse opals are fcc structures of close-packed air spheres in a solid matrix of $\mathrm{TiO}_{2}$. Details of the preparation and characterization of the opals and inverse opals can be found in Ref. [22]. We have studied four polystyrene samples with lattice parameters $a=178 \pm 3$ and $365 \pm 5 \mathrm{~nm}$ and eight titania samples with lattice parameters $a=370,420,500,580$, and $650 \pm 10 \mathrm{~nm}$. All samples have typical dimensions of $2 \times 2 \times 0.2 \mathrm{~mm}^{3}$ and contain highquality domains with diameters larger than $50 \mu \mathrm{m}$. These domains have flat faces normal to the 111 crystal axis, which is evident from scanning electron microscope images. The other crystalline axes are randomly oriented.

The polystyrene opals were doped with the laser dye Rhodamine $6 \mathrm{G}(\mathrm{R} 6 \mathrm{G})$ by soaking them for $30 \mathrm{~min}$ in a dilute solution $\left(10^{-6} \mathrm{~mol} / \mathrm{l}\right)$ of the dye in ethanol. Afterward the samples were rinsed in ethanol and dried to remove the dye from the sample surface. To estimate an upper bound to the density of the dye, we assume that the infiltrating solution completely fills the air voids in the opals, and that, in the process of drying, the dye molecules distribute uniformly on the surfaces of the spheres inside the opals. Knowing the volume of the air voids in each unit cell of the opals $\left(0.26 a^{3}\right)$ and the dye concentration, we arrive at no more than ten dye molecules per unit-cell inner surface [23] (for opals with lattice parameter $a=365 \mathrm{~nm}$ ). While this surface density increases linearly with the lattice parameter $a$, the average distance between the dye molecules remains more than two orders of magnitude away from the typical intermolecular distances where reabsorption and energy-transfer processes could play a role [24]. Before emission experiments, we performed a selective bleaching [25] of the dye at the surface of opal photonic crystals in order to ensure that the emission is recorded only from the bulk of the crystal, and not from the crystal surface [13]. The whole surface was illuminated for up to $1 \mathrm{~h}$ by an intense laser beam at the Bragg angle for the pump frequency. At this angle the pump intensity decreases exponentially with depth, which implies that the dye

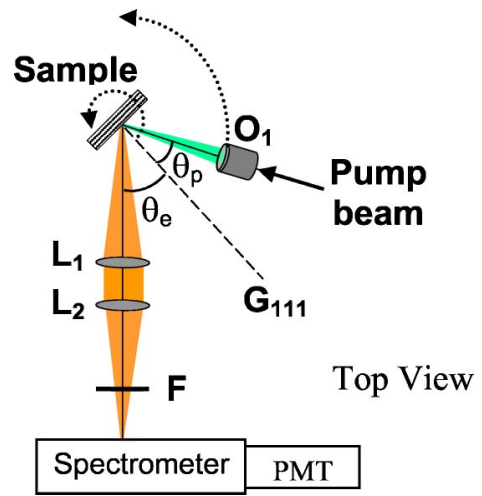

FIG. 1. (Color online) Scheme of the experimental setup. The pump beam is focused onto the sample at incident angle $\theta_{p}$ by the objective $O_{1}(f=7.3 \mathrm{~cm}$, numerical aperture 0.05$)$. Luminescence within a cone centered at detection angle $\theta_{e}$ relative to the surface normal is collected by the lens $L_{1}(f=12 \mathrm{~cm})$ and imaged on the spectrometer slit by the lens $L_{2}(f=12 \mathrm{~cm})$. A color filter $F$ prevents scattered pump light from entering the prism spectrometer. The angle $\theta_{e}$ is varied by rotating the rotation stage, which carries the sample holder, the fiber, and the objective $O_{1}$, whereas the incident angle $\theta_{p}$ is kept fixed.

bleaches within the first few crystal layers. However, our experimental results were found to be almost independent of the amount of bleaching, thus indicating that emitters in the bulk of the photonic crystals provide the dominating contribution to the measured emission intensity.

The titania inverse opals were infiltrated for $24 \mathrm{~h}$ with a colloidal suspension of $\mathrm{ZnSe}$-coated $\mathrm{CdSe}$ quantum dots $[26,27]$ in a mixture of $50 \%$ chloroform and $50 \%$ butanol. Afterward the samples were rinsed in chloroform and dried. The concentration of the infiltrating solution was $10^{-7} \mathrm{~mol} / \mathrm{l}$. We used the same estimation in order to get the maximum number of quantum dots per unit cell in the inverse opals. For samples with the largest lattice parameter $a=650 \mathrm{~nm}$, this concentration is 15 quantum dots per unit cell and is sufficiently low to avoid energy-transfer processes and reabsorption. In order to minimize oxidation and contamination of the quantum dots, the inverse opals were infiltrated with the quantum dots in a nitrogen-purged glove box and held in a sealed chamber under a 1.7 mbar nitrogen atmosphere during the optical measurements. No bleaching at the surface layers could be performed for the quantum dots, however a detailed analysis of the angular emission data (see below) demonstrates that light emission from the quantum dots from the bulk dominates.

\section{B. Experimental setup}

Figure 1 shows the experimental setup used to measure emission from light sources inside photonic crystals. The sources inside the crystal are excited by a cw Ar-ion laser $(\lambda=497 \mathrm{~nm})$ with the power at the sample around $10 \mu \mathrm{W}$. At this pump power, we do not observe any effects of bleaching of the dye during the emission experiments. To focus the pump beam on the sample surface a fiber-coupled microscope objective is used. The beam is focused to a spot of 
about $30 \mu \mathrm{m}$ in diameter at an incident angle $\theta_{p}$ relative to the surface normal, usually $\theta_{p} \approx 25^{\circ}$. In order to acquire emission spectra as a function of the detection angle $\theta_{e}$ relative to the surface normal, the sample is mounted on a rotation stage. The surface normal is parallel to the 111 reciprocal lattice vector $G_{111}$. In order to illuminate the same area irrespective of $\theta_{e}$, the fiber-coupled objective $\left(O_{1}\right)$ is mounted on the same rotation stage as the sample. In this way, the angle of incidence $\theta_{p}$ between the pump beam and the surface normal is kept constant. The advantage over previous experiments, where the sample was rotated with respect to both the pump and detection beams, is that the absolute intensity of the angle-dependent spectra can be reliably compared. The position of the pump spot on the sample is monitored with a microscope. The emitted light is collected within a cone of $15^{\circ}$ full width around the angle $\theta_{e}$, imaged on the slit of the spectrometer with $4 \mathrm{~nm}$ resolution, and detected by the photomultiplier tube (PMT). The angleresolved spectra are usually measured at the detection angles from $0^{\circ}$ to $75^{\circ}$ at intervals of $15^{\circ}$. The measured spectra are corrected for the dark count of the PMT. The shapes of the spectra are confirmed to be independent of the pump intensity, and the emitted intensity is linear with the pump power.

\section{DIFFUSE LIGHT TRANSPORT IN PHOTONIC CRYSTALS}

\section{A. Escape function}

In real photonic structures, defects in the arrangement of the building blocks are always present and cause random multiple scattering of light. This means that all light emitted in such photonic structures becomes diffuse on length scales equal to the transport mean free path $l$, which is often much smaller than the thickness of the sample $L$. For example, our opals and inverse opals have mean free paths of about $15 \mu \mathrm{m}$ [28], whereas the thickness of the samples is about $200 \mu \mathrm{m}$. Thus, even though photons generated inside a photonic crystal are diffracted by the crystal structure, this effect is smeared out by the random multiple scattering while the photons propagate through the bulk towards the crystal surface. Only at distances from the surface $z$ smaller than $l$, where the photons emanate ballistically toward the crystalair interface after a last scattering event, the effect of Bragg diffraction is not destroyed by the scattering. Hence the diffuse emission acquires a directional dependence only when it exits the crystal [21].

We consider the ratio of the mean free path $l$ to the attenuation length for Bragg diffraction $L_{B}$ in order to estimate the attenuation of emission caused by Bragg diffraction, as proposed in Ref. [15]. Since the mean free path $l$ is larger than the Bragg attenuation length $L_{B}$ (typically $l / L_{B} \sim 2-5$ [28]), an attenuation in the stop band equal to $1-L_{B} / l$ $=50 \%$ to $80 \%$ is predicted, which is in agreement with our observations. As will be discussed later, the stop-band attenuations are obtained directly from reflectivity measurements; therefore, the mean free path $l$ is not an explicit parameter in our theoretical model.

In the present work we investigate directional properties of light emitted by sources from 3D photonic crystals and compare to a model of diffuse light transmission through opaque media [29-31] extended to photonic crystals [21]. Based on the diffusion theory, the intensity of light $I\left(\omega, \mu_{e}\right)$ with frequency $\omega$ that exits the sample at external angles between $\theta_{e}=\cos ^{-1}\left(\mu_{e}\right)$ and $\cos ^{-1}\left(\mu_{e}+d \mu_{e}\right)$ relative to the surface normal is equal to

$$
I\left(\omega, \mu_{e}\right) d \mu_{e}=I_{\text {tot }}(\omega) P\left(\omega, \mu_{e}\right) d \mu_{e} .
$$

Here $I_{\text {tot }}(\omega)$ is the total spontaneous emission power that is the spectrum of the light sources integrated over the exit angles $\theta_{e}$. For sources with a low quantum efficiency or with inhomogeneously broadened spectra, $I_{\text {tot }}(\omega)$ is proportional to the LDOS [9]. The distribution $P\left(\omega, \mu_{e}\right)$ is defined as

$$
P\left(\omega, \mu_{e}\right)=\mu_{e} \frac{n_{e}^{2}}{n_{i}^{2}}\left(\frac{1+\bar{R}(\omega)}{1-\bar{R}(\omega)}+\frac{3}{2} \mu_{i}\right)\left[1-R\left(\omega, \mu_{i}\right)\right],
$$

where $n_{e}$ and $n_{i}$ are average refractive indices outside and inside the sample [32], respectively. $\mu_{e}$ and $\mu_{i}$ are related by Snell's law. $R\left(\omega, \mu_{i}\right)$ is an angle-dependent internalreflection coefficient that yields an angle-averaged internalreflection coefficient $\bar{R}(\omega)$ :

$$
\begin{gathered}
\bar{R}(\omega)=\frac{3 C_{2}(\omega)+2 C_{1}(\omega)}{3 C_{2}(\omega)-2 C_{1}(\omega)+2}, \\
C_{n}(\omega)=\int_{0}^{1} R\left(\omega, \mu_{i}\right) \mu_{i}^{n} d \mu_{i} .
\end{gathered}
$$

From the diffusion theory, $\bar{R}(\omega)$ determines the so-called extrapolation length that sets the boundary conditions of the diffuse intensity [29-31]. The normalized function $P\left(\omega, \mu_{e}\right)$ describes the distribution of emission intensity over the escape angles and will be called the "escape function." In absence of reflection effects, the escape function tends to the well-known Lambertian distribution of diffuse surfaces.

In random media such as powders or macroporous sponges the internal-reflection coefficient $R\left(\omega, \mu_{i}\right)$ is barely frequency dependent [33], and propagation through the interface is well described by Fresnel reflection model assuming an average refractive index. The angular dependence of the escape function $P\left(\omega, \mu_{e}\right)$ agrees well with experiments on random media [30,31]. For highly dispersive photonic crystals, however, the Fresnel model cannot describe the internal reflection since light escaping from a depth $z<l$ from the crystal surface is Bragg attenuated for angles and frequencies inside a stop band. We model the strong angle- and frequency-dependent internal reflection with photonic band structures. At a particular frequency $\omega^{*}$ where a stop band is present, the internal reflectivity $R\left(\omega^{*}, \mu_{i}\right)$ blocks the emission in the directions of the stop band (a range of $\mu_{i}$ 's related to $\omega^{*}$ by the photonic band structure) and therefore reduces the escape function $P\left(\omega^{*}, \mu_{e}\right)$, [cf. Eq. (2)]. The presence of the stop band raises the angle-integrated reflectivity $\bar{R}\left(\omega^{*}\right)$, which enhances the escape function $P\left(\omega^{*}, \mu_{e}\right)$ for angles outside the stop band. Thus, the escape function is strongly nonLambertian in a photonic crystal, showing clear suppressions or enhancements. 

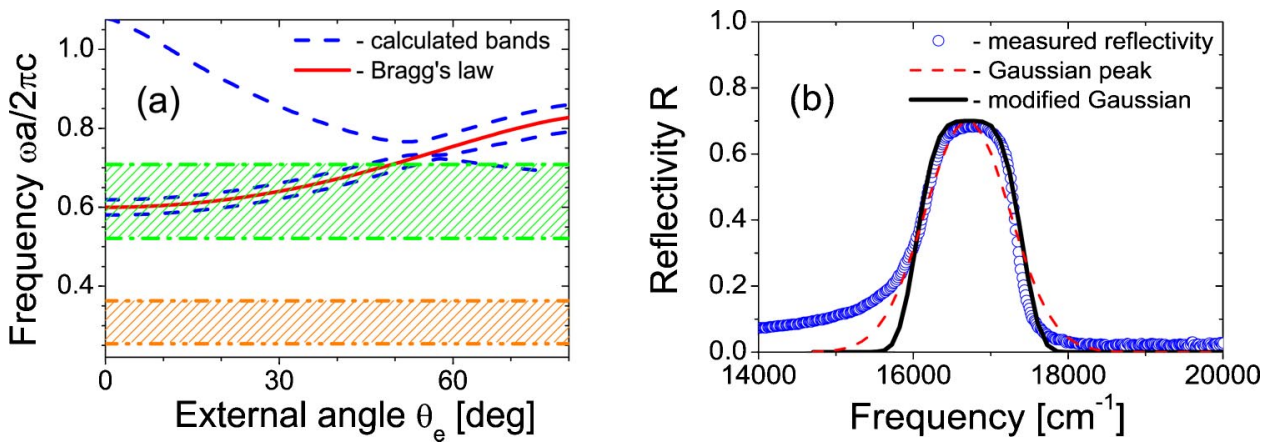

FIG. 2. (Color online) (a) Photonic band structure for polystyrene opals [(blue) dashed curves], center frequency of the stop band vs detection angle $\theta_{e}$ according to Bragg's law [(red) solid curve], and the frequency range of R6G emission for opals with lattice parameters $a=178$ and $365 \mathrm{~nm}$ [hatched regions between the (orange) dash-dot-dotted lines and the (green) dash-dotted lines, respectively]. (b) Normal-incidence reflectivity as a function of frequency for polystyrene opals with a lattice parameter $a=365 \mathrm{~nm}$ and refractive-index contrast $m=1.59$. (Blue) circles are measured values, the (black) solid curve is a fit with the modified Gaussian reflectivity peak [Eq. (5)], the (red) dashed curve is a fit with a Gaussian reflectivity peak.

\section{B. Internal-reflection coefficient}

In order to model the internal-reflection coefficient $R\left(\omega, \mu_{i}\right)$, we have taken into consideration calculated photonic band structures. Figure 2(a) shows the photonic band structure for polystyrene opals calculated along the $L U$ and $L K$ lines in reciprocal space in the frequency range around the first-order stop gap ( $L$-gap) that is due to optical Bragg diffraction by (111) planes parallel to the sample surface. The emission spectrum of R6G is in the low-frequency limit relative to the stop bands of opals with a lattice parameter $a=178 \mathrm{~nm}$ [region confined by the (orange) dash-dot-dotted lines]. Consequently, this sample is effectively homogeneous for the emission frequencies, and therefore it can serve as a reliable reference. For these nonphotonic crystals we used the Fresnel model in order to describe the internal reflection. In Fig. 2(a) one can also see the frequency gap between the two lowest bands [(blue) dashed curves], which obeys Bragg's law [solid (red) curve] within the frequency range of R6G emission for the opals with a lattice parameter $a$ $=365 \mathrm{~nm}$ [region confined by the (green) dash-dotted lines]. Therefore, the angular dependence of the center frequency of the $L$ gap is modeled with the solid (red) curve, i.e., $\omega_{C}\left(\mu_{i}\right)=\omega_{C}\left(\mu_{i}=1\right) / \mu_{i}$. To investigate the frequency dependence of the reflectivity, we have performed reflectivity experiments on the samples using external incident plane waves, since this technique reveals the center frequencies and the widths of stop bands [20,34]. Figure 2(b) shows a normal-incidence reflectivity spectrum measured from an opal with the lattice parameter $a=365 \mathrm{~nm}$ [(blue) circles]. The reflectivity peak is not fitted well with a Gaussian [(red) dashed curve]. As an improved model, we propose a modified Gaussian [Fig. 2(b), solid (black) curve]:

$$
R_{1}\left(\omega, \mu_{i}\right)=A_{1}\left(\mu_{i}\right) \exp \left[-\frac{\left[\omega-\omega_{C}\left(\mu_{i}\right)\right]^{4}}{2\left[\Delta \omega_{C}\left(\mu_{i}\right)\right]^{4}}\right]
$$

where $A_{1}\left(\mu_{i}\right)$ is the magnitude of the internal-reflection coefficient and $\Delta \omega_{C}\left(\mu_{i}\right)$ is the width parameter. This peak shape is seen to fit the measurements well for frequencies $>16000 \mathrm{~cm}^{-1}$. At frequencies below the stop band, i.e., be- low $16000 \mathrm{~cm}^{-1}$ for these particular samples with $a$ $=365 \mathrm{~nm}$, a deviation from the model is observed. We attribute this deviation to Fresnel reflection, which is important only in the low-frequency limit and therefore is not relevant for the escape function of photonic samples. The width $\Delta \omega_{C}\left(\mu_{i}\right)$ hardly varies with $\mu_{i}$ within the range of the dye emission; therefore it is taken to be constant in our model. The magnitude of the internal-reflection coefficient $A_{1}\left(\mu_{i}\right)$ decreases with $\mu_{i}$ because at larger internal angles $\theta_{i}$ $=\cos ^{-1}\left(\mu_{i}\right)$ the path length for the light to become Bragg attenuated increases with $\mu_{i}$, and this increases the probability of scattering at $z<L_{B}$. The value of $A_{1}\left(\mu_{i}\right)$ at $\mu_{i}=1$ is taken from the normal-incidence reflectivity experiments. Thus, we have $A_{1}\left(\mu_{i}\right)=A_{1}\left(\mu_{i}=1\right) \mu_{i}$ and $A_{1}\left(\mu_{i}=1\right)=0.7$ [see Fig. 2(b)].

Emitted light that is scattered within a distance $L_{B}<z$ $<l$ toward the exit interface can also be redirected by Bragg diffraction by the sets of $(11 \overline{1})$ planes, which are oriented at $\theta_{i}=70.5^{\circ}$ to the $(111)$ planes and the surface normal. The internal-reflection coefficient $R_{2}\left(\omega, \mu_{i}\right)$ for Bragg diffraction by $(11 \overline{1})$ lattice planes is modeled similarly to $R_{1}\left(\omega, \mu_{i}\right)$. Taking into account that we measure emission from many randomly oriented crystal domains in azimuthal directions, the reflectivity $R_{2}\left(\omega, \theta_{i}\right)$ is averaged over the azimuthal angles $\phi$ between the $L K$ and $L U$ lines in reciprocal space, yielding

$$
R_{2}\left(\omega, \theta_{i}\right)=\frac{3}{\pi} \int_{0}^{\pi / 3} A_{2}\left(\theta_{i}, \phi\right) \exp \left[-\frac{\left[\omega-\omega_{C}\left(\theta_{i}, \phi\right)\right]^{4}}{2\left[\Delta \omega_{C}\left(\theta_{i}\right)\right]^{4}}\right] d \phi .
$$

The magnitude $A_{2}\left(\theta_{i}, \phi\right)$ is modeled as $A_{2}\left(\theta_{i}, \phi\right)$ $=A_{2}\left(70.5^{\circ}, 0^{\circ}\right) \cos \left(\theta_{i}-70.5^{\circ}\right) \cos (\phi)$ with $A_{2}\left(70.5^{\circ}, 0^{\circ}\right)$ $=0.7$. The total-internal-reflection coefficient $R\left(\omega, \mu_{i}\right)$ is calculated as a sum of the $R_{1}\left(\omega, \mu_{i}\right)$ and $R_{2}\left(\omega, \cos \left(\theta_{i}-70.5^{\circ}\right)\right)$ modified Gaussian peaks. We expect this model of the angleand frequency-dependent internal reflectivity to capture the essential frequency dependence of the first-order photonic stop bands in polystyrene opals. 

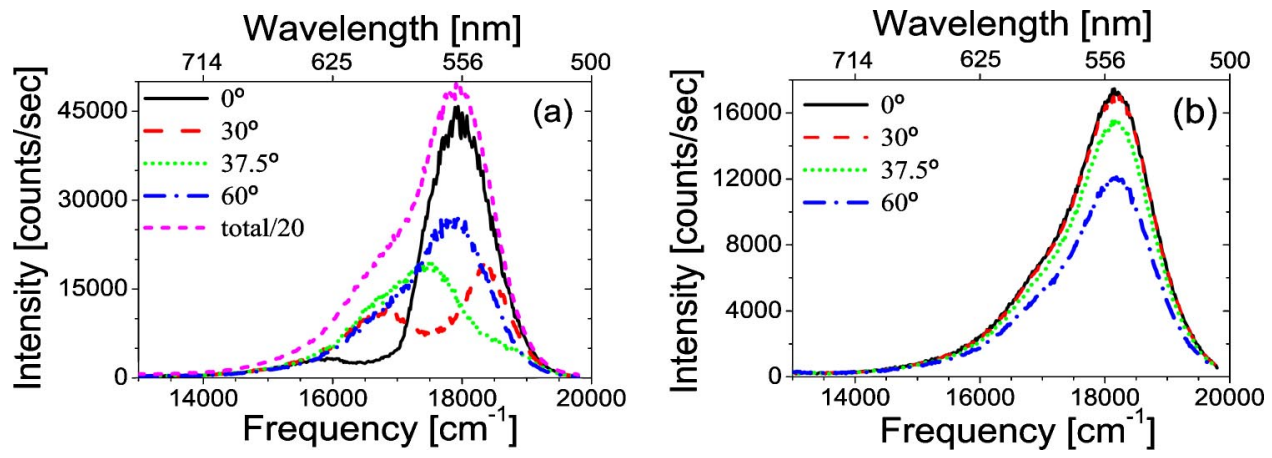

FIG. 3. (Color online) Emission spectra of R6G in polystyrene opals with lattice parameters $a=365$ (a) and $178 \mathrm{~nm}$ (b). The (black) solid curves are obtained at $\theta_{e}=0^{\circ}$, the (red) dashed curves at $\theta_{e}=30^{\circ}$, the (green) dotted curves at $\theta_{e}=37.5^{\circ}$, and the (blue) dash-dotted curves at $\theta_{e}=60^{\circ}$. The (magenta) short-dashed curve in (a) indicates the total emission spectrum $I_{\text {tot }}(\omega)$.

In the case of the titania inverse opals we apply the same escape model to explain our experimental data. However, in calculating the internal-reflection coefficient $R\left(\omega, \mu_{i}\right)$, Bragg diffraction from other lattice planes must also be included. This difference compared to polystyrene opals appears since titania inverse opals are more strongly photonic and the measurements were performed at higher reduced frequencies $(a / \lambda=\omega a / 2 \pi c)$. Moreover, the resulting stop bands occur at lower detection angles $\theta_{e}$ in these crystals than in the polystyrene opals, as a consequence of the lower average refractive index. Therefore Bragg's law is not a sufficient approximation and is not used to model the angular dependence of the stop bands in the titania inverse opals. Instead, the full band structure model is employed, in which we take into account multiple Bragg wave coupling $[15,36]$. For the inverse opals, this model was already successfully tested on diffuse transmission experiments [21].

\section{RESULTS AND DISCUSSION}

\section{A. Spontaneous emission of R6G in polystyrene opals}

Reflectivity measurements at normal incidence of polystyrene opals [Fig. 2(b)] reveal that the relative width of the first-order stop band is $\Delta \omega / \omega \approx 0.075$. For opals with a lattice parameter $a=365 \mathrm{~nm}$ this means a stop band in the range $16100-17300 \mathrm{~cm}^{-1}$ for light escaping the crystal normally to the surface. The dye R6G emits in the range of $15000-20000 \mathrm{~cm}^{-1}$, and hence we expect to observe direction-dependent emission of the dye from the opals with $a=365 \mathrm{~nm}$. Figure 3(a) displays the emission spectra at selected detection angles for such doped opals. It is clearly seen that the shapes of the spectra are affected by the photonic crystal. The emission is suppressed by the crystal for $\theta_{e}$ $=0^{\circ}$ in the spectral range from 16000 to $17500 \mathrm{~cm}^{-1}$. With increasing angle $\theta_{e}$ the low-frequency parts of the emission recover, and the suppressed emission range shifts to higher frequencies, as expected from Bragg's law for a photonic stop band from a single set of lattice planes. In contrast, the shape of the spectra from an opal with a lattice parameter $a=178 \mathrm{~nm}$ remains unchanged [Fig. 3(b)]. The sample is not photonic for the frequency range considered: the frequencies of R6G emission lie far below the first-order stop band in the opal with this lattice parameter.
Before studying spontaneous emission from photonic samples we have verified the applicability of the abovementioned model of diffuse light transport on the nonphotonic, reference samples. We use Fresnel reflection to describe the angular-dependent internal-reflection coefficient, taking an average refractive index $n_{\mathrm{av}}=1.44$, which is derived from the polystyrene filling fraction $\varphi \approx 74 \%$ in opals and the refractive index of polystyrene $n=1.59$. We record the intensity at the maximum of the emission spectrum as a function of the exit angle $\theta_{e}$ relative to the measurement at $\theta_{e}=0^{\circ}$. The relative intensity $I\left(\theta_{e}\right)$ is compared to the escape function $P\left(\theta_{e}\right)$ in Fig. 4. While the expected decrease with angle is observed, it is clear that the calculated intensity differs systematically from the measured data. This deviation appears to be caused by an angle-dependent detection efficiency as a result of an increase with $\theta_{e}$ of the projection of the spectrometer slit on the sample. Correcting the measured intensity $I\left(\omega, \mu_{e}\right)$ for the detection efficiency $D\left(\mu_{e}\right)$ (see the Appendix) yields the corrected intensity $I_{c}\left(\omega, \mu_{e}\right)$ $=I\left(\omega, \mu_{e}\right) / D\left(\mu_{e}\right)$ displayed as stars in Fig. 4. The agreement between the corrected intensity and the calculated escape function is excellent. With the proper account of the detec-

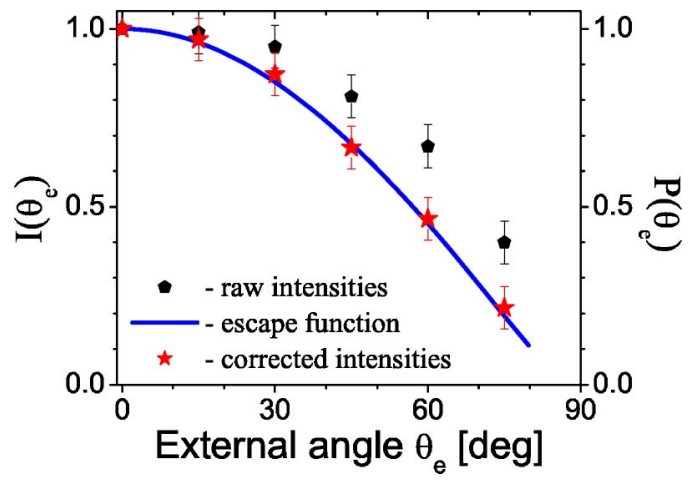

FIG. 4. (Color online) Angular distribution of the R6G emission from a polystyrene opal with a lattice parameter $a=178 \mathrm{~nm}$. The (black) pentagons indicate the intensity measured at the spectral maximum $\left(\omega=17860 \mathrm{~cm}^{-1}\right.$ or $\left.\lambda=560 \mathrm{~nm}\right)$; the (blue) solid curve is the calculated escape function with Fresnel internal-reflection coefficient. Measured intensities corrected for the detection efficiency of the setup are displayed as (red) stars. All data are normalized at $\theta_{e}=0^{\circ}$. 


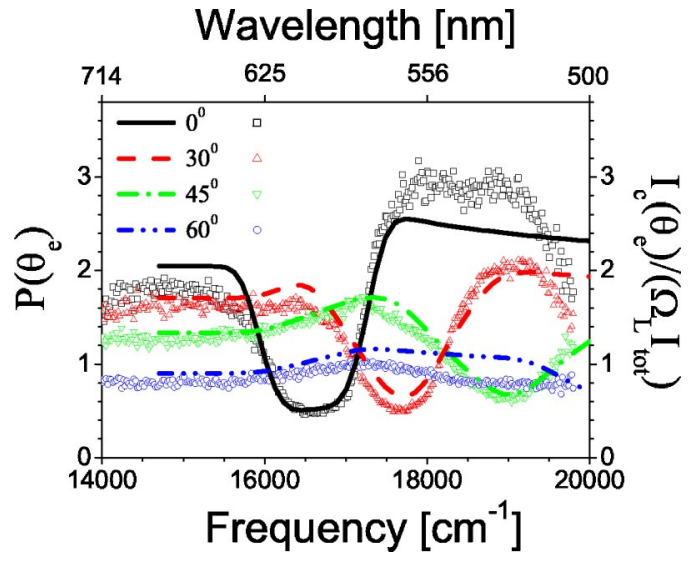

FIG. 5. (Color online) R6G emission from polystyrene opal with lattice parameter $a=365 \mathrm{~nm}$. The scatter plots represent the measured spectra corrected for the angular aperture of the collecting lens $\Omega_{L}$ and divided by the total emission spectrum $I_{\text {tot }}(\omega)$. The calculated escape functions are plotted with solid curves. The (black) solid curve and squares are for $\theta_{e}=0^{\circ}$, (red) dashed curve and triangles are for $\theta_{e}=30^{\circ}$, (green) inverted triangles and solid curve are for $\theta_{e}=45^{\circ}$, and (blue) circles with dash-dotted curve are for $\theta_{e}=60^{\circ}$.

tion efficiency, the angular distribution of emission escaping the reference samples is thus fully understood. In all experimental data presented in the remainder of this paper the detection efficiency has been included.

In the case of the photonic samples the exit distribution of emission strongly depends on the frequency $\omega$ as mentioned above: $P\left(\omega, \theta_{e}\right)=I_{c}\left(\omega, \theta_{e}\right) / I_{\text {tot }}(\omega)$. The total emission spectrum $I_{\text {tot }}(\omega)$ is determined by discretely summing the angleresolved spectra $I_{c}\left(\omega, \theta_{e}\right)$ weighted by $2 \pi \sin \left(\theta_{e}\right) d \theta_{e}$ to approximate the integration over $2 \pi$ solid angle. The spectra from Fig. 3(a) divided by the total emission spectrum $I_{\text {tot }}(\omega)$ are plotted in Fig. 5 (symbols) together with the calculated escape function $P\left(\omega, \theta_{e}\right)$ (curves). We observe a good agreement between our experiment and theory. The escape function hardly varies with frequency in the low-frequency region $\leqslant 15600 \mathrm{~cm}^{-1}$, while it still depends on the detection direction. In contrast, at higher frequencies strong variations are seen compared to the low-frequency range. At the exit angle $\theta_{e}=0^{\circ}$, the escape function is significantly reduced in the spectral range from 16000 to $17500 \mathrm{~cm}^{-1}$ by the stop band centered at $\omega=16700 \mathrm{~cm}^{-1}$ due to internal Bragg diffraction, which is described by the term $\left[1-R\left(\omega, \mu_{i}\right)\right]$ in Eq. (2). The change of the center frequency as well as the decrease in the attenuation of emission inside the stop band with increasing exit angle $\theta_{e}$ are well described in our model by the frequency- and angular-dependent internal-reflection coefficient $R\left(\omega, \mu_{i}\right)$. At $\theta_{e}=60^{\circ}$, the stop band has moved out of the spectral range of R6G.

Figure 5 also shows a peculiar feature: the frequency ranges where the emission is inhibited along certain directions adjoin with the ranges where emission is increased along the same directions. Such an increase appears at the blue side of the stop band at $\theta_{e}=0^{\circ}$ and $30^{\circ}$, and at the red side of the stop band at $\theta_{e}=45^{\circ}$ and $60^{\circ}$. This enhanced escape probability in the frequency range 16000-19500

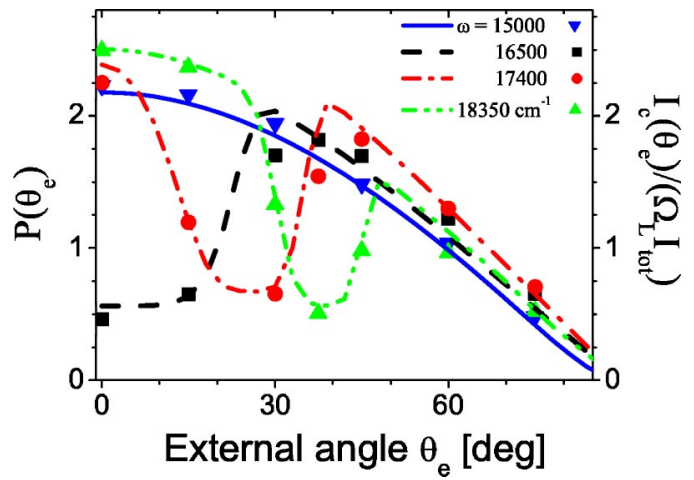

FIG. 6. (Color online) Intensity ratios $I_{c}\left(\theta_{e}\right) / I_{\text {tot }}$ corrected for the lens aperture $\Omega_{L}$ as a function of the exit angle $\theta_{e}$ for a polystyrene opal with a lattice parameter $a=365 \mathrm{~nm}$ for frequencies $\omega$ $=15000,16500,17400$, and $18350 \mathrm{~cm}^{-1}$ [(blue) inverted triangles, (black) squares, (red) circles, and (green) triangles]. The corresponding curves represent the calculated escape distributions (for reduced frequencies $\omega a / 2 \pi c=0.55,0.6,0.64$, and 0.67, respectively).

$\mathrm{cm}^{-1}$ along directions that do not coincide with the stop band is reflected in our model for $P\left(\omega, \mu_{e}\right)$ [Eq. (2)] as an increase of the angle-averaged internal-reflection coefficient $\bar{R}(\omega)$. The good agreement between experiments and theory confirms a qualitative attribution of such enhancements to diffuse escape effects [18]. Moreover it unambiguously demonstrates that our experimental observation of the emission enhancement is not due to Bragg standing wave effects proposed in Ref. [35], but is related to diffusion of light.

In Fig. 6 we compare the experimentally determined intensity distributions (symbols) for several fixed frequencies with the calculated ones (solid curves) as functions of the exit angle $\theta_{e}$. The experimental values of $P\left(\theta_{e}\right)$ were obtained by dividing the emission $I_{c}\left(\theta_{e}\right)$ by the total emission spectrum $I_{\text {tot }}$ and correcting for the angular aperture of the collecting lens $\Omega_{L}$. For the frequency $\omega=15000 \mathrm{~cm}^{-1}$, below the stop band, the distribution follows the Lambertian distribution and is similar to the exit distribution from the nonphotonic sample (Fig. 4). For the frequencies above the red edge of the stop band we observe strongly non-Lambertian behavior. For the frequency $\omega=16500 \mathrm{~cm}^{-1}$ emission is suppressed relative to the Lambertian distribution in the range of the exit angles from $\theta_{e}=0^{\circ}$ to $20^{\circ}$. This range moves to larger exit angles for the frequency $\omega=17400 \mathrm{~cm}^{-1}$ in qualitative agreement with Bragg's law. For $\omega=18300 \mathrm{~cm}^{-1}$ the suppression observed around $\theta_{e}=40^{\circ}$ is preceded by a considerable increase of emission in the angle range $0^{\circ}$ to $20^{\circ}$. A qualitative explanation of this effect is as follows. Some escape directions are blocked by internal Bragg diffraction, and diffusion eventually distributes this back-reflected light along the remaining directions. Thus, light is more likely to exit the crystal along these allowed directions. From Figs. 5 and 6 we conclude that the escape function is in excellent agreement with the measured angle-dependent spectra. To the best of our knowledge, the current work provides the first quantitative modeling of spontaneous emission spectra in 3D photonic crystals. 


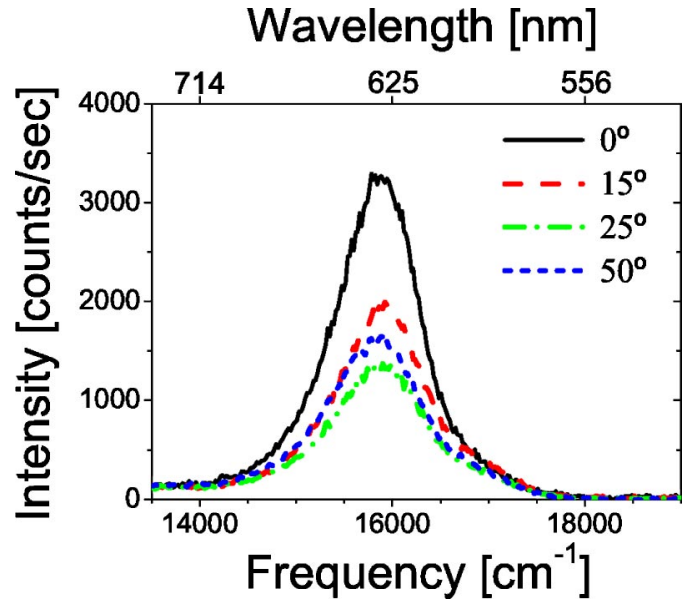

FIG. 7. (Color online) Emission spectra of CdSe quantum dots in a titania inverse opal with a lattice parameter $a=500 \mathrm{~nm}$. The (black) solid curve is obtained at $\theta_{e}=0^{\circ}$, the (red) dashed curve at $\theta_{e}=15^{\circ}$, the (green) dash-dotted curve at $\theta_{e}=25^{\circ}$, and the (blue) short-dashed curve at $\theta_{e}=50^{\circ}$.

\section{B. Spontaneous emission from quantum dots in titania inverse opals}

Titania inverse opals possess a larger relative width of the first-order stop band $(\Delta \omega / \omega \approx 0.16)$ than the polystyrene opals owing to their inverse structure and high refractiveindex contrast $(m=2.7)$. The concomitant large modifications of the LDOS makes the inverse opals very attractive for control of propagation and spontaneous emission of light $[9,10]$. Figure 7 shows emission spectra of CdSe quantum dots in a titania inverse opal with lattice parameter $a=500 \mathrm{~nm}$ for selected detection angles $\theta_{e}$. No significant changes in the spectral shapes due to internal Bragg diffraction are observed, because the relative spectral width of the light sources $(\Delta \omega / \omega<0.06)$ is considerably smaller than the width of the stop band of the photonic crystal. This shows that the escape distribution $P\left(\omega, \theta_{e}\right)$ does not vary significantly within the frequency range of the quantum dot spec- trum. In contrast, a strong angular dependence of the emission intensity is apparent in Fig. 7. As a consequence, effects of Bragg diffraction are most convincingly observed by recording the angular dependencies at the spectral maxima of the emission spectra.

In Fig. 8(a) we present escape distributions from titania inverse opals with lattice parameters $a=370$ and $420 \mathrm{~nm}$ at frequency $\omega=16300 \mathrm{~cm}^{-1}$, and with a lattice parameter $a$ $=500 \mathrm{~nm}$ at $\omega=15870 \mathrm{~cm}^{-1}$. Both measured (symbols) and calculated (curves) values are shown. For the crystal with the lattice parameter $a=370 \mathrm{~nm}$, for which the center frequency of the quantum-dot spectrum lies below the stop band, the escape function follows the Lambertian distribution. A large deviation from the Lambertian distribution is observed for the quantum dot emission from the crystals with the other two lattice parameters. In the crystals with $a=420 \mathrm{~nm}$, the emission is strongly reduced in the range of the angles from $\theta_{e}=0^{\circ}$ to $35^{\circ}$, and it is enhanced at higher exit angles. For the crystals with $a=500 \mathrm{~nm}$, the suppression is shifted to the range of $\theta_{e}=20^{\circ}$ to $45^{\circ}$, as expected for photonic gaps at higher reduced frequency $(a / \lambda=\omega a / 2 \pi c)$, and in excellent agreement with our theoretical predictions. The stop-band ranges are noticeably wider than that in the case of the polystyrene opals (Fig. 6), which is due to a wider frequency range of the stop band in the titania inverse opals. To the best of our knowledge, this is the first demonstration of photonic crystal bands in the emission spectra of confined excitons in quantum dots.

Figure 8(b) shows the photonic band structure for a titania inverse opal. The hatched regions indicate the stop band caused by Bragg diffraction by (111) lattice planes. In the angular range from $\theta_{e}=35^{\circ}$ to $55^{\circ}$, multiple Bragg wave coupling from (111) and (200) diffracted waves takes place $[15,36]$. The horizontal bars represent the reduced center frequencies of the quantum dot emission from the inverse opals with the lattice parameters $a=370,420,500,580$, and $650 \mathrm{~nm}$. The colors of the bars indicate the measured values of the escape function $P\left(\omega, \theta_{e}\right)$. For reduced frequencies around the stop bands, it is seen that inhibited escape probability appears in the angular ranges of the stop bands,
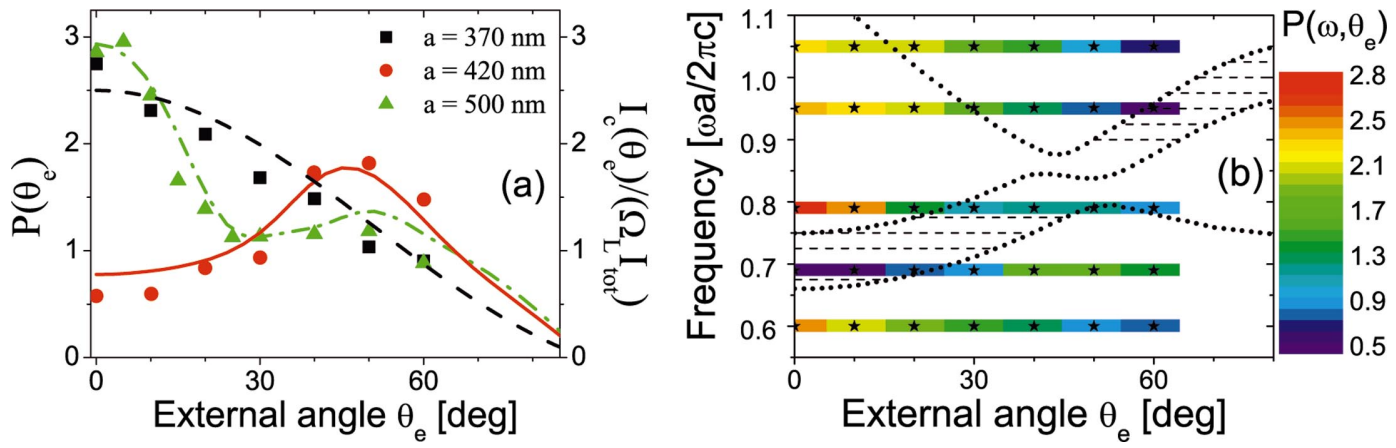

FIG. 8. (Color) (a) Escape distribution as a function of the exit angle $\theta_{e}$ for titania inverse opals with lattice parameters $a=370$ and $420 \mathrm{~nm}$ at $\omega=16390 \mathrm{~cm}^{-1}(\lambda=610 \mathrm{~nm}$, black squares and red circles, respectively), and with a lattice parameter $a=500 \mathrm{~nm}$ at $\omega$ $=15870 \mathrm{~cm}^{-1}$ ( $\lambda=630 \mathrm{~nm}$, green triangles). The corresponding curves represent the calculated distributions. (b) Photonic band structure for the inverse opals (black dotted curves). The hatched regions indicate the stop band caused by Bragg diffraction by (111) lattice planes. The horizontal bars represent the reduced center frequencies of the quantum-dot emission from the crystals with lattice parameters (bottom to top) $a=370,420,500,580$, and $650 \mathrm{~nm}$. The colors of the bars indicate the values of the escape function $P\left(\omega, \theta_{e}\right)$ obtained from the measurements at exit angles shown by the black stars. 


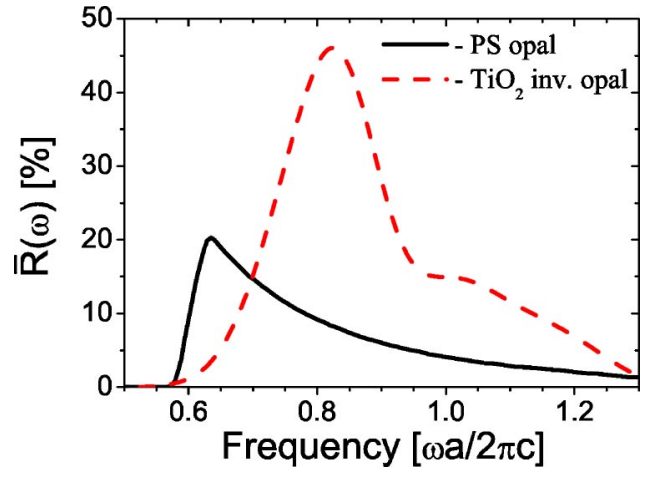

FIG. 9. (Color online) The angle-averaged internal reflectivity $\bar{R}(\omega)$ for the polystyrene opals [(black) solid curve] and for the titania inverse opals [(red) dashed curve] according to the diffusion model, in which only the first-order Bragg diffraction is taken into account. $\bar{R}(\omega)$ determines the enhancement of the escape probability outside a stop-band direction.

whereas enhanced escape is found outside the stop bands. Hence, the photonic crystals are seen to "funnel" light along certain allowed directions.

For experiments on quantum-dot emission in photonic crystals, the good agreement between the experimentally obtained escape distributions and the calculated ones confirms that the light emanating from inside the crystals is diffuse. It also confirms that the observed emission is dominated by sources inside the bulk of the crystal. We can exclude that light sources on the sample surface contribute significantly: their emission would give rise to an angle-independent component of the intensity that is not observed. Furthermore, observation of stop bands in emission spectra is important for successful lifetime experiments or other quantum-optical studies of light sources in photonic crystals. The stop bands are evidence that the emission from the light sources is strongly coupled to the photonic crystals, and are a prerequisite for time-resolved experiments of changes in the emission decay rate caused by a modified LDOS [10].

Based on the close accordance of the experiments with the model, we can extract the angle-averaged internalreflection coefficient $\bar{R}(\omega)$. Figure 9 shows that $\bar{R}(\omega)$ is as large as $50 \%$ for the titania inverse opals and up to $20 \%$ for the polystyrene opals. The internal-reflection coefficient varies strongly with frequency in contrast to the frequencyindependent $\bar{R}$ in random media. The coefficient increases with solid angle for Bragg reflection, starting from the lowfrequency edge of the $L$ gap. For the opals, the maximum $\bar{R}$ occurs at the high-frequency edge of the $L$ gap, where the reflecting stop bands extend over the largest solid angle [34]. For the inverse opals, the maximum $\bar{R}$ occurs at higher reduced frequencies in the range of multiple Bragg wave coupling $(\omega a / 2 \pi c \sim 0.85)[15]$. The shoulder near 1.0 is attributed to the inclusion of (200) reflection condition in our model. In a more elaborate escape model with additional diffraction conditions, we may expect additional peaks in the angle-averaged reflection coefficient at even higher frequencies. Since the inverse opals interact stronger with light than the opals, their stop bands are wider and hence the angle averaged reflectivity is larger, in agreement with our observations.

A closer consideration of the reflectivity coefficients can serve to optimize the spontaneous emission yield of light sources (atoms, dyes, or quantum dots) embedded inside thick photonic crystals $(L>l)$. Such an optimization can be achieved either via the excitation of the sources, via their emission, or both. First, the excitation efficiency can be increased by realizing that increased escape probability also implies an increased probability for excitation light to enter a photonic crystal. Thus, by tuning an excitation beam to frequencies and angles of high escape, the combined action of diffusion and Bragg diffraction retains relatively more excitation light inside the sample, thus increasing the probability for spontaneous emission of the embedded light sources. Second, spontaneously emitted radiation is efficiently channeled out of the sample along particular directions. This occurs when the lattice parameter of the photonic crystal is chosen such that the emission frequencies are in the range of enhanced $\bar{R}(\omega)$. A clear example of enhanced escape is apparent in Fig. 5 at $\theta_{e}=0^{\circ}$ near $18000 \mathrm{~cm}^{-1}$. In the ultimate case of a photonic band gap, it has even been predicted that the diffuse emission is extremely directional; see Ref. [21]. Third, one can envision situations where both excitation and emission are enhanced: in Fig. 8(b) enhanced escape probability occurs both at $\omega a / 2 \pi c \sim 0.8$ and $\theta_{e}=0^{\circ}$ and at $\omega a / 2 \pi c \sim 0.7$ and $\theta_{e}=50^{\circ}$. Thus, by tuning the excitation to the former condition and the emission to the latter, the spontaneous emission yield is expected to be enhanced by at least a factor of 2. Further improvements should be feasible in photonic crystals with even larger $\bar{R}$.

\section{CONCLUSIONS}

We have presented experimental data on angular-resolved emission from light sources embedded in efficient 3D photonic crystals. The experiments were compared in detail to a recently developed model of light transport in real photonic crystals that are influenced by disorder. Our model is based on diffusion of light due to scattering (disorder) combined with angle- and frequency-dependent internal reflections (order). Good quantitative agreement between experiment and theory confirms that the details of the emission spectra are determined by the intricate interplay of order and disorder. Properties of the stop bands, such as their frequency range, magnitude, and angular dependence, are extracted from the experiment by analyzing the emission escape function. The enhanced escape probability for emission along directions outside the stop bands is explained by the angle-averaged internal-reflection coefficient $\bar{R}(\omega)$. The diffuse and angulardependent nature of light escaping from the photonic crystals proves that the light comes from emitters inside the crystals. By measuring the escape functions of the quantum-dot emission from the titania inverse opals, we have revealed clear stop bands in the quantum-dot emission spectra, confirming that the confined excitons experience optical confinement. The quantitative agreement between experiment and theory 
demonstrates that light propagation and spontaneous emission in real 3D photonic crystals is well understood.

\section{ACKNOWLEDGMENTS}

We thank Floris van Driel and Daniël Vanmaekelbergh (University of Utrecht) for preparation of the quantum dots, Léon Woldering for photonic crystal preparation, Arie Irman and Karin Overgaag for experimental assistance, and last but not least Femius Koenderink for band-structure calculations and discussions on escape functions. This work is a part of the research program of the Stichting voor Fundamenteel Onderzoek der Materie (FOM), which is financially supported by the Nederlandse Organisatie voor Wetenschappelijk Onderzoek (NWO).

\section{APPENDIX: MODEL OF DETECTION EFFICIENCY OF EMISSION SET-UP}

The aim of this appendix is to explain the difference between the calculated escape distribution and the measured intensity illustrated in Fig. 4. This difference appears because the width of the projection of the collection optics (the spectrometer slit) on the sample increases with the angle $\theta_{e}$. The sample surface is placed in the focus of the collecting lens $L_{1}$, the spectrometer slit is in the focus of the imaging lens $L_{2}$ (cf. Fig. 1). The only emission collected emanates from the region confined by the slit projection on the sample surface, see Fig. 10. This means that the spectrometer collects light from a larger region on the surface at larger detection angles, and that the measured angle-dependent intensity should be corrected for the detection efficiency of the setup. The detection efficiency is modeled as a ratio $D\left(\mu_{e}\right)$ of the intensity $B\left(\mu_{e}\right)$ collected from the surface region within the slit projection (Fig. 10) at detection angle $\theta_{e}=\cos ^{-1}\left(\mu_{e}\right)$ to the intensity $B\left(\mu_{e}=1\right)$ collected at normal angle:

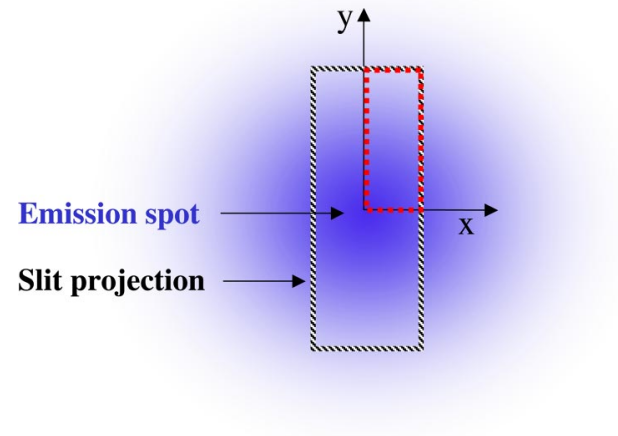

FIG. 10. (Color online) Real-space cartoon of the projection of the spectrometer slit (striped rectangle) on the sample surface overlapped with the emission spot (blue). The quarter of the slit projection [(red) dashed rectangle] has one of the corners in the emissionspot center. The width of the projection (along $x$ ) increases at larger detection angles $\theta_{e}$.

$$
D\left(\mu_{e}\right)=\frac{B\left(\mu_{e}\right)}{B\left(\mu_{e}=1\right)}, \quad B\left(\mu_{e}\right)=\int_{0}^{x\left(\mu_{e}\right)} \int_{0}^{y_{0}} I(x, y) d x d y .
$$

We take into account that the integration runs over a quarter of the slit, as $x\left(\mu_{e}\right)=x_{0} / \mu_{e}$ is the half-width of the slit projection, and $x_{0}$ and $y_{0}$ are the half-width and the half-height of the slit projection at $\mu_{e}=\cos \left(\theta_{e}\right)=1$, respectively. Typical values of $x_{0}$ and $y_{0}$ in the experiments are $50 \mu \mathrm{m}$ and $1 \mathrm{~mm}$. It is assumed that the intensity of diffuse light on the sample surface around the pump beam varies as $I(r) \propto l^{2} /\left(l^{2}+r^{2}\right)$, where $r^{2}=x^{2}+y^{2}$ is the distance from the axis of the pump beam along the sample surface, and $l$ is the mean free path of light in the sample.
[1] Photonic Crystals and Light Localization in the 21st Century, edited by C. M. Soukoulis (Kluwer, Dordrecht, 2001).

[2] V. P. Bykov, Sov. J. Quantum Electron. 4, 861 (1975).

[3] E. Yablonovitch, Phys. Rev. Lett. 58, 2059 (1987).

[4] S. John, Phys. Rev. Lett. 58, 2486 (1987).

[5] N. Vats, S. John, and K. Busch, Phys. Rev. A 65, 043808 (2002).

[6] Y. Yang, M. Fleischhauer, and S.-Y. Zhu, Phys. Rev. A 68, 043805 (2003).

[7] R. Sprik, B. A. van Tiggelen, and A. Lagendijk, Europhys. Lett. 35, 265 (1996).

[8] K. Busch and S. John, Phys. Rev. E 58, 3896 (1998).

[9] A. F. Koenderink, L. Bechger, H. P. Schriemer, A. Lagendijk, and W. L. Vos, Phys. Rev. Lett. 88, 143903 (2002).

[10] P. Lodahl, A. F. van Driel, I. S. Nikolaev, A. Irman, K. Overgaag, D. Vanmaekelbergh, and W. L. Vos, Nature (London) 430, 654 (2004).

[11] V. N. Bogomolov, S. V. Gaponenko, I. N. Germanenko, A. M. Kapitonov, E. P. Petrov, N. V. Gaponenko, A. V. Prokofiev, A.
N. Ponyavina, N. I. Silvanovich, and S. M. Samoilovich, Phys. Rev. E 55, 7619 (1997).

[12] A. Blanco, C. Lòpez, R. Mayoral, H. Miguez, F. Meseguer, A. Mifsud, and J. Herrero, Appl. Phys. Lett. 73, 1781 (1998).

[13] M. Megens, J. E. G. J. Wijnhoven, A. Lagendijk, and W. L. Vos, J. Opt. Soc. Am. B 16, 1403 (1999).

[14] Y. A. Vlasov, M. Deutsch, and D. J. Norris, Appl. Phys. Lett. 76, 1627 (2000).

[15] H. P. Schriemer, H. M. van Driel, A. F. Koenderink, and W. L. Vos, Phys. Rev. A 63, 011801(R) (2001).

[16] Y. Lin, J. Zhang, E. H. Sargent, and E. Kumacheva, Appl. Phys. Lett. 81, 3134 (2002).

[17] A. F. Koenderink, A. Lagendijk, and W. L. Vos, e-print physics/0406052.

[18] L. Bechger, Ph.D. thesis, University of Twente, 2003 (ISBN: 90-365-1991-8); L. Bechger, P. Lodahl, and W. L. Vos, J. Phys. Chem. B (unpublished).

[19] A. F. Koenderink, P. M. Johnson, J. F. Galisteo Lòpez, and W. L. Vos, C. R. Physique 3, 67 (2002). 
[20] J. F. Galisteo-Lòpez, E. Palacios-Lidòn, E. Castillo-Martìnez, and C. Lòpez, Phys. Rev. B 68, 115109 (2003).

[21] A. F. Koenderink and W. L. Vos, Phys. Rev. Lett. 91, 213902 (2003); J. Opt. Soc. Am. B 22, 1075 (2005).

[22] J. E. G. J. Wijnhoven, L. Bechger, and W. L. Vos, Chem. Mater. 13, 4486 (2001).

[23] In a fcc structure consisting of spheres of radius $r$ and having a lattice parameter $a$ equal to $\sqrt{8} r$, the inner surface per unit cell $A$ equals $4 \times 4 \pi r^{2}=2 \pi a^{2}$. Therefore the surface density $N_{s}$ of the dye is proportional to $0.26 a^{3} / 2 \pi a^{2} \sim a$.

[24] J. R. Lakowicz, Principles of Fluorescence Spectroscopy, 2nd ed. (Kluwer, New York, 1999).

[25] Bleaching is a photoinduced damage of dye molecules (fluorophores). Typically, it happens because of oxidation of the fluorophore that damages the molecule permanently.

[26] B. O. Dabbousi, J. Rodriguez-Viejo, F. V. Mikulec, J. R. Heine, H. Mattoussi, R. Ober, K. F. Jensen, and M. G. Bawendi, J. Phys. Chem. B 101, 9463 (1997).

[27] C. de Mello Donega, S. G. Hickey, S. F. Wuister, D. Vanmaekelbergh, and A. Meijerink, J. Phys. Chem. B 107, 489
(2003).

[28] A. F. Koenderink, M. Megens, G. van Soest, W. L. Vos, and A. Lagendijk, Phys. Lett. A 268, 104 (2000).

[29] A. Lagendijk, R. Vreeker, and P. de Vries, Phys. Lett. A 136, 81 (1989).

[30] J. X. Zhu, D. J. Pine, and D. A. Weitz, Phys. Rev. A 44, 3948 (1991).

[31] D. J. Durian, Phys. Rev. E 50, 857 (1994).

[32] The average refractive index of a photonic crystal is estimated as $n_{\text {av }}=\varphi n_{m}+(1-\varphi) n_{\text {air }}$, where $\varphi$ is the volume fraction of the solid material, and $n_{m}$ and $n_{\text {air }}$ are refractive indices of the solid material and air, respectively.

[33] F. J. P. Schuurmans, D. Vanmaekelbergh, J. van de Lagemaat, and A. Lagendijk, Science 284, 141 (1999).

[34] M. S. Thijssen, R. Sprik, J. E. G. J. Wijnhoven, M. Megens, T. Narayanan, A. Lagendijk, and W. L. Vos, Phys. Rev. Lett. 83, 2730 (1999).

[35] A. G. Galstyan, M. E. Raikh, and Z. V. Vardeny, Phys. Rev. B 62, 1780 (2000).

[36] H. M. van Driel and W. L. Vos, Phys. Rev. B 62, 9872 (2000). 